\title{
Novel lymphocyte to red blood cell ratio (LRR), neutrophil to red blood cell ratio (NRR), monocyte to red blood cell ratio (MRR) as predictive and prognostic biomarkers for locally advanced breast cancer
}

\author{
Yaohui Wang", Haofeng Wang", Wenjin Yin, Yanping Lin, Liheng Zhou, Xiaonan Sheng, Yaqian Xu, Rui Sha, \\ Jinsong Lu \\ Department of Breast Surgery, Renji Hospital, School of Medicine, Shanghai Jiaotong University, Shanghai 200127, China \\ Contributions: (I) Conception and design: Y Wang, J Lu; (II) Administrative support: W Yin, J Lu; (III) Provision of study materials or patients: All \\ authors; (IV) Collection and assembly of data: All authors; (V) Data analysis and interpretation: All authors; (VI) Manuscript writing: All authors; (VII) \\ Final approval of manuscript: All authors \\ "These authors contributed equally to this work. \\ Correspondence to: Jinsong Lu, MD. Department of Breast Surgery, Renji Hospital, School of Medicine, Shanghai Jiaotong University, No. 1630 \\ Dongfang Road, Shanghai 200127, China. Email: lujjss@163.com.
}

\begin{abstract}
Background: Lymphocytes, neutrophils, and monocytes are vital effector cells in innate immunity. We postulated that lymphocyte to red blood cell ratio (LRR), neutrophil to red blood cell ratio (NRR), monocyte to red blood cell ratio (MRR) could represent the intensity of systemic inflammatory immunological reaction reflected through the lymphocyte, neutrophil and monocyte respectively. This study aimed to access the predictive and prognostic value of LRR, NRR, MRR and LRR-NRR-MRR score for locally advanced breast cancer.

Methods: A total of 137 patients from two clinical trials SHPD002 and SHPD003 were included. Logistic regression analysis was used to evaluate the association between ratios and pathological complete response (pCR). Disease-free survival (DFS) and overall survival (OS) were estimated by Kaplan-Meier method and cox regression analysis.

Results: Lower LRR-NRR-MRR score (OR $=0.593$; 95\% CI: 0.369-0.954; $\mathrm{P}=0.031$ ) was more easily to achieve pCR in multivariate analysis. Lower LRR $(\mathrm{P}=0.022)$, NRR $(\mathrm{P}=0.027)$ and MRR $(\mathrm{P}=0.024)$ were significantly associated with better DFS. LRR-NRR-MRR score was an independently prognostic factor for both DFS (HR =3.318; 95\% CI: 1.601-6.876; P=0.001) and OS (HR =3.160; 95\% CI: 1.030-9.696; $\mathrm{P}=0.044)$.

Conclusions: The LRR-NRR-MRR score could be identified as a new predictive biomarker for the therapeutic effect of neoadjuvant therapy and an independent prognostic factor for both DFS and OS for locally advanced breast cancer.
\end{abstract}

Keywords: Lymphocytes; neutrophils; monocytes; red blood cell; predictive; prognosis

Submitted Aug 09, 2019. Accepted for publication Oct 08, 2019.

doi: $10.21037 /$ gs.2019.10.10

View this article at: http://dx.doi.org/10.21037/gs.2019.10.10 


\section{Introduction}

Breast cancer is the most frequently diagnosed cancer among women all over the world (1). Patients who are initially diagnosed at locally advanced stage need neoadjuvant therapy (2). Nowadays, neoadjuvant therapy is increasingly common and has more meaning including reducing clinical stage to facilitate surgery and even more improving the long-time clinical outcome (3). Exploring biomarkers to predict the sensitivity of neoadjuvant therapy and the prognosis of those patients attracts more and more attentions.

It is well-established that the response to anticancer therapies and prognosis of cancer was not only associated with histopathological characteristics of the tumor itself, but also the host, such as inflammatory and immunological response (4). Lymphocytes, neutrophils, and monocytes are vital effector cells in innate immunity and known to play a critical role in various inflammatory status. The pretreatment neutrophil to lymphocyte ratio (NLR) $(5,6)$ and lymphocyte to monocyte ratio (LMR) (7) were reported to be related with responses to neoadjuvant chemotherapy. Low NLR and high LMR were supposed to be favorable prognostic factors in breast cancer patients with neoadjuvant chemotherapy $(8,9)$.

The reverse change of the numerator and denominator of ratio like NLR could reflect obvious variation of lymphocytes and neutrophils. However, the aligned change of specific cells might make the ratio constant. Thus, those ratios reported previously insufficiently reflected the real inflammatory immune status of the body. In this study, we hypothesized that the red blood cell could be used as a conference parameter and generated the new ratios. We postulated that lymphocyte to red blood cell ratio (LRR), neutrophil to red blood cell ratio (NRR), monocyte to red blood cell ratio (MRR) could represent the intensity of systemic inflammatory immunological reaction reflected through the lymphocyte, neutrophil and monocyte in the peripheral blood respectively. We try to analysis the predictive and prognostic effect of these ratios and a combined LRR, NRR, MRR (LRR-NRR-MRR) score in locally advanced breast cancer patients received neoadjuvant therapy.

\section{Methods}

\section{Patients and treatment}

Patients included in this study were from two clinical trials SHPD002 (NCT02221999) and SHPD003 (NCT02879513) from Oct, 2013 to Nov, 2016. All patients had signed informed consent. All procedures were complied with the ethical standards of institutions and national research committees, as well as the 1964 Helsinki Declaration. The study was permitted by Institutional Review Board (IRB) of Renji Hospital, School of Medicine, Shanghai Jiao Tong University (IRB approval number: [2017]088). The follow-up of database was prospectively. All patients were histologically confirmed locally advanced breast cancer with no distant metastasis (cT size $\geq 2 \mathrm{~cm}$ or $\mathrm{cN} \geq 1$ ). The main eligibility criteria were having comprehensive record of whole blood test before the first neoadjuvant therapy and completing the surgery after the neoadjuvant therapy.

Patients received paclitaxel $80 \mathrm{mg} / \mathrm{m}^{2}$ on day $1,8,15,22$ every 28 days for four cycles and cisplatin $25 \mathrm{mg} / \mathrm{m}^{2}$ on day $1,8,15$ every 28 days for four cycles. The human epidermal growth factor receptor 2 (HER2) positive patients were recommended using trastuzumab concurrent with neoadjuvant and lasting for 1 year. In the SHPD002, the hormone receptor positive patients were randomly received neoadjuvant chemotherapy concurrent with endocrine therapy or not. In the neoadjuvant chemotherapy combined with endocrine therapy group, the postmenopausal patients received aromatase inhibitor and premenopausal patients received gonadotropin releasing hormone agonist. After the four cycles neoadjuvant therapy, patients received the surgery. Adjuvant chemotherapy was randomly given with two cycles of weekly paclitaxel, cisplatin or four cycles of cyclophosphamide, epirubicin and fluorouracil, if the patients didn't achieve the pathological complete response (pCR). Radiotherapy was delivered according to the radiologist. Hormone receptor positive patients received the adjuvant endocrine therapy.

\section{Data collection}

Clinical characteristic of patients, including age, menstruation, body mass index (BMI), stage of tumor, pathological biopsy information of tumor before neoadjuvant therapy and pCR conditions were collected. The definition of estrogen receptor (ER)/progesterone receptor $(\mathrm{PR})$ positive was more than $1 \%$ stained cells. HER2 positive was defined as immuno-histochemistry $3+$ or amplification of the HER2 gene (FISH positive). The pCR definition was the absence of invasive tumor in the breast and no pathological involvement of axillary lymph nodes at 
time of surgery.

Peripheral whole blood test was obtained immediately after clinical diagnosis of breast cancer and before neoadjuvant therapy. LRR was defined as absolute lymphocyte count divided by absolute red blood cell count. NRR was defined as absolute neutrophil count divided by absolute red blood cell count. MRR was defined as the absolute monocyte count divided by absolute red blood cell count.

\section{Statistical methods}

We assessed the correlation between clinical characteristics and the pretreatment LRR, NRR and MRR by using Wilcoxon rank sum test. The association between the LRR, NRR, MRR, all kinds of clinical characteristics, and pCR were calculated by univariate, multivariate logistic regression analysis. Disease-free survival (DFS) was defined as the time from surgery until the first occurrence of locoregional relapse, distant metastasis, contralateral breast cancer, other second primary cancer or death from any cause. Overall survival (OS) was recorded the date from surgery to death from any cause. Patients who remained alive and event-free at their date of last follow-up were censored. The Kaplan-Meier method and log-rank test were used for univariate survival analysis. The multivariate survival analysis was estimated with the cox proportional regression hazards model. The multivariate adjusted factors for both multivariate logistic regression analysis and survival analysis were same, including clinical $\mathrm{T}$ stage (T1 T2 or T3 T4), ER status of biopsy (negative or positive), PR status of biopsy (negative or positive), ki67 index of biopsy ( $\leq 20$ or $>20$ ), HER2 status (negative $v s$. positive) of biopsy and BMI ( $<23.5, \geq 23.5)$ before the neoadjuvant therapy. The MaxStat analysis was used to identify the optimally dichotomously cut-off point for LRR, NRR and MRR in the survival analysis. The cut-off points were $0.384,1.17$, 0.0853 for LRR, NRR and MRR, respectively. According to the result of survival analysis, poor prognostic factors were labeled as score of 1 and good prognostic factors were labeled as score of 0 . Thus, high LRR, NRR, MRR were assigned a score of 1 and low LRR, NRR, MRR were assigned a score of 0 . LRR-NRR-MRR score of one patient were the sum score of LRR, NRR and MRR. The patient was divided into four group according to the LRR-NRRMRR score $(0,1,2,3)$. All $\mathrm{P}$ values were two sided. $\mathrm{P}$ value $<0.05$ was considered statistically significant for all results. The STATA 14.0 (Stata Corporation, College Station, TX,
USA) and statistical package $\mathrm{R}$ (version 3.3.2, R Project for Statistical Computing, https://www.r-project.org/) were used for statistical analyses.

\section{Results}

\section{General clinical characteristics}

There were 139 patients included in this study. General clinical characteristics were shown in Table 1 . The median follow-up was 24 months (IQR, 15-36 months). Forty of 139 patients (28.78\%) achieved pCR. LRR and MRR had no significant difference in all clinical characteristics. The lower NRR was found in the patients with HER2 positive group $(\mathrm{P}=0.033)$.

\section{Association of $L R R, N R R, M R R, L R R-N R R-M R R$ score and $p C R$}

In the univariate analysis, there was no significant association between LRR, NRR or MRR and pCR. However, LRR-NRR-MRR score significantly predicted pCR $(\mathrm{OR}=0.661 ; 95 \% \mathrm{CI}: 0.443-0.986 ; \mathrm{P}=0.042)$. Compared with score of 2,3 , patients with score of 0 , 1 were more easily to achieved pCR (Figure 1). At the meantime, the lower ER ( $\mathrm{P}=0.000)$, lower PR $(\mathrm{P}=0.049)$, higher Ki67 $(\mathrm{P}=0.002)$, HER2 positive $(\mathrm{P}=0.002)$ and lower $\mathrm{BMI}(\mathrm{P}=0.026)$ did favor for pCR (Table S1).

In the multivariate analysis, $\mathrm{LRR}(\mathrm{OR}=0.239 ; 95 \% \mathrm{CI}$ : $0.085-0.672 ; \mathrm{P}=0.007)$ was significantly associated with pCR. However, NRR $(\mathrm{P}=0.268)$ and MRR $(\mathrm{P}=0.527)$ were not detected the association with pCR. Meanwhile, the lower LRR-NRR-MRR score significantly favored pCR $(\mathrm{OR}=0.593 ;$ 95\% CI: 0.369-0.954; $\mathrm{P}=0.031$, Table S2).

In the hormone receptor positive and HER2 positive subgroup, LRR-NRR-MRR score obviously associated with pCR (OR $=0.451 ; 95 \%$ CI: 0.233-0.871; P=0.018, Table S3, Figure 1).

\section{Survival analysis}

In the univariate analysis, DFS ( $\mathrm{P}=0.010$, Figure 2) was significantly higher in the LRR-low group. But there was no significant difference of DFS between the NRR-low and NRR-high group in the univariate analysis $(\mathrm{P}=0.362$, Figure 2). In the multivariate analysis, the $\mathrm{LRR}(\mathrm{HR}=4.447$; 95\% CI: $1.236-16.00 ; \mathrm{P}=0.022$, Table S4) and the NRR (HR $=4.524 ; 95 \%$ CI: $1.191-17.18 ; \mathrm{P}=0.027$, Table S5) were 
Table 1 Correlations between LRR, NRR, MRR and clinicopathological characteristics of all patients

\begin{tabular}{|c|c|c|c|c|c|c|c|c|c|c|}
\hline Variables & $\begin{array}{c}\text { Total } \\
\text { patients } \\
(\mathrm{N}=139)\end{array}$ & \multicolumn{3}{|c|}{ LRR } & \multicolumn{3}{|c|}{ NRR } & \multicolumn{3}{|c|}{ MRR } \\
\hline \multicolumn{11}{|l|}{ Age, years } \\
\hline$\leq 50$ & 66 & 26 & 40 & 0.489 & 52 & 14 & 0.029 & 30 & 36 & 0.271 \\
\hline \multicolumn{11}{|l|}{ Menopausal status } \\
\hline Premenopausal & 64 & 25 & 39 & 0.456 & 49 & 15 & 0.005 & 28 & 36 & 0.150 \\
\hline Postmenopausal & 75 & 34 & 41 & & 70 & 5 & & 42 & 33 & \\
\hline \multicolumn{11}{|l|}{ Clinical T stage } \\
\hline No & 22 & 8 & 14 & 0.529 & 21 & 1 & 0.152 & 12 & 10 & 0.669 \\
\hline Yes & 117 & 51 & 66 & & 98 & 19 & & 58 & 59 & \\
\hline \multicolumn{11}{|l|}{ ER status } \\
\hline Negative & 39 & 17 & 22 & 0.865 & 35 & 4 & 0.386 & 21 & 18 & 0.608 \\
\hline Positive & 100 & 42 & 58 & & 84 & 16 & & 49 & 51 & \\
\hline \multicolumn{11}{|l|}{ PR status } \\
\hline \multicolumn{11}{|l|}{ Ki67 index } \\
\hline$\leq 20$ & 34 & 15 & 19 & 0.821 & 27 & 7 & 0.236 & 17 & 17 & 0.962 \\
\hline$>20$ & 105 & 44 & 61 & & 92 & 13 & & 53 & 52 & \\
\hline \multicolumn{11}{|l|}{ BMI index } \\
\hline$<23.5$ & 73 & 29 & 44 & 0.495 & 61 & 12 & 0.469 & 40 & 33 & 0.271 \\
\hline$\geq 23.5$ & 66 & 30 & 36 & & 58 & 8 & & 30 & 36 & \\
\hline
\end{tabular}

HER2, human epidermal growth factor receptor 2; ER, Estrogen receptors; PR, Progesterone receptors; LRR, lymphocyte to red blood cell ratio; NRR, neutrophil to red blood cell ratio; MRR, monocyte to red blood cell ratio; BMI, body mass index.

independent prognostic factors for DFS. In addition, both the LRR-low group ( $\mathrm{P}=0.025$, Figure 3$)$ and the NRR-low group $(\mathrm{P}=0.025$, Figure 3$)$ achieved better OS.

Meanwhile, result was found significantly in favor of MRR-low group for DFS ( $\mathrm{P}=0.047$, Figure 2), not for
OS $(\mathrm{P}=0.453$, Figure 3$)$ in the univariate analysis. In the multivariate analysis, the lower MRR was also associated with better DFS (HR $=3.940 ; 95 \%$ CI: 1.201-12.93; $\mathrm{P}=0.024)$, not for OS (HR $=2.196$; $95 \% \mathrm{CI}$ : 0.352-13.72; $\mathrm{P}=0.400$, Table S6). 


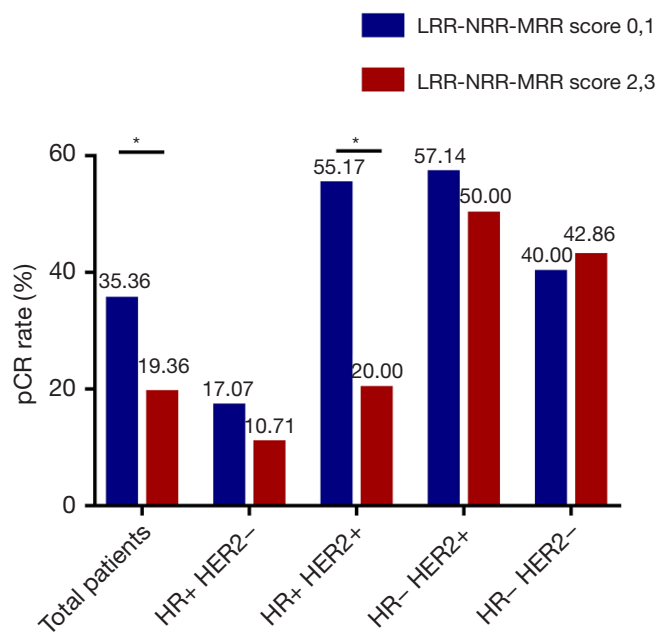

Figure 1 The pCR rates in all subtype breast cancer patients according to the LRR-NRR-MRR score. ${ }^{*}, \mathrm{P}<0.05$. pCR, pathological complete response; LRR, lymphocyte to red blood cell ratio; NRR, neutrophil to red blood cell ratio; MRR, monocyte to red blood cell ratio.
Altogether, the LRR-NRR-MRR score was shown the good prognosis in the lower score group for DFS $(\mathrm{P}=0.040$, Figure 2) and marginally for $\mathrm{OS}(\mathrm{P}=0.087$, Figure 3$)$ in the univariate analysis. In the multivariate analysis, LRR-NRRMRR score was the independently prognostic factor for both DFS (HR $=3.318 ; 95 \%$ CI: 1.601-6.876; $\mathrm{P}=0.001$ ) and OS (HR $=3.160 ; 95 \%$ CI: 1.030-9.696; $\mathrm{P}=0.044$, Table 2).

In the hormone receptor positive subgroup, LRR-NRRMRR score was an independent prognostic factor for DFS (HR $=3.518 ; 95 \%$ CI: $1.575-7.860 ; \mathrm{P}=0.002)$ and $\mathrm{OS}$ (HR $=5.882 ; 95 \%$ CI: $1.004-34.47 ; \mathrm{P}=0.050)$. Similarly, in the HER2 negative subgroup, LRR-NRR-MRR score was also the independent prognostic factors for DFS $(\mathrm{HR}=4.608$; 95\% CI: $1.446-14.68 ; \mathrm{P}=0.010$ ) but not for the OS (HR $=4.531 ; 95 \%$ CI: $0.728-28.22 ; \mathrm{P}=0.105)$.

\section{Discussion}

Lymphocyte, monocyte and neutrophil were increasingly found playing vital roles in carcinogenesis and prognosis of
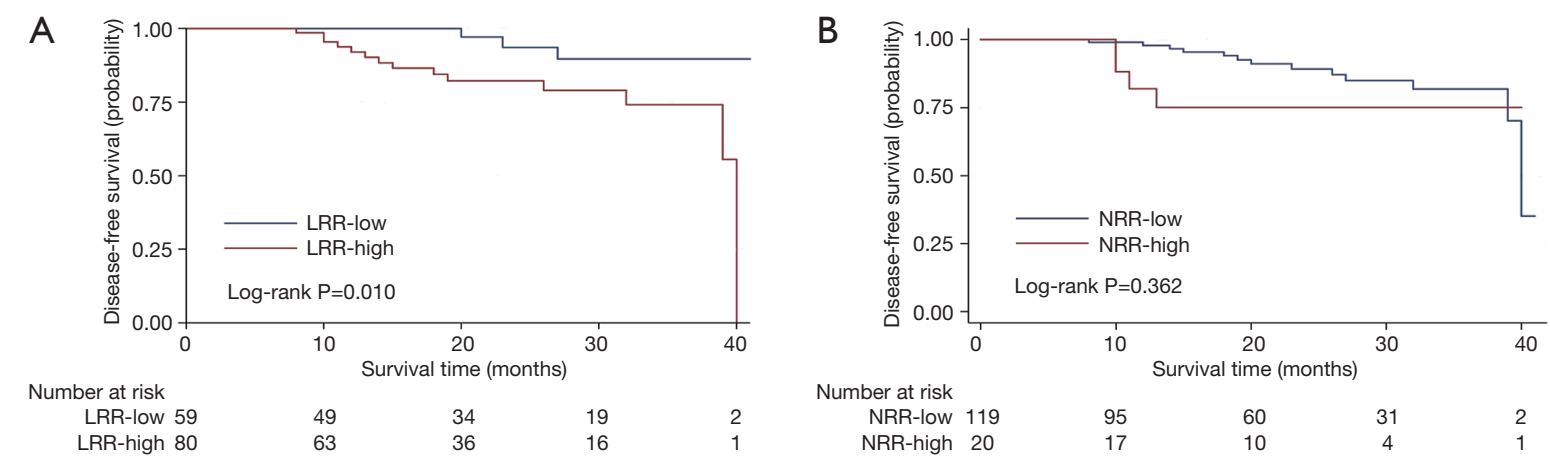
LRR-high 80
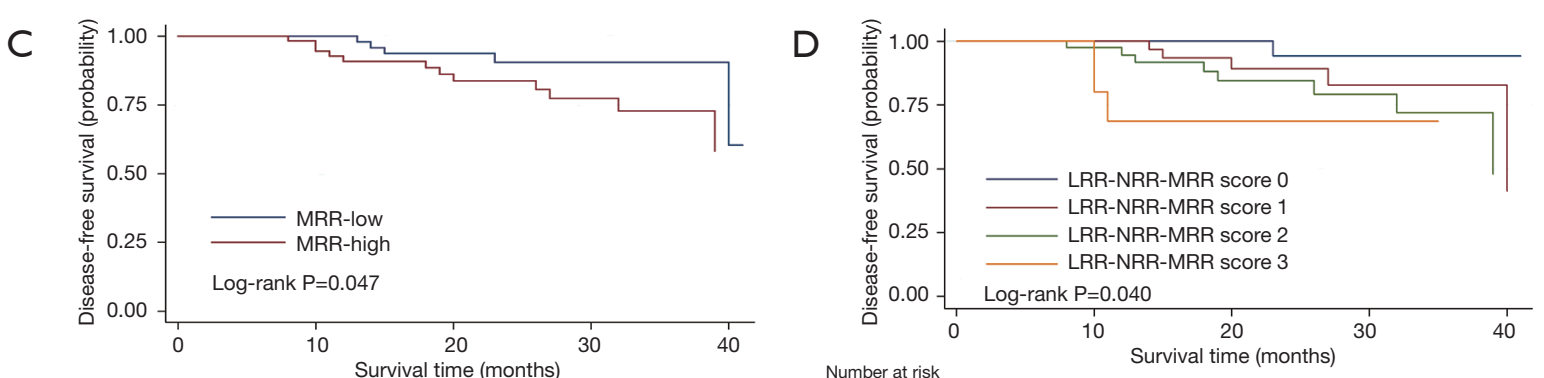

58
54

35
35

18
17
LRR-NRR-MRR score 039

LRR-NRR-MRR score 143

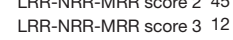

$\begin{array}{lccl}33 & 21 & 13 & 1 \\ 34 & 22 & 9 & 2 \\ 35 & 22 & 11 & 0 \\ 10 & 5 & 2 & 0\end{array}$

Figure 2 The Kaplan-Meier survival curve for disease-free survival in the locally advanced breast cancer patients. (A) Analysis by LRR; (B) analysis by NRR; (C) analysis by MRR; (D) analysis by the LRR-NRR-MRR score. LRR, lymphocyte to red blood cell ratio; NRR, neutrophil to red blood cell ratio; MRR, monocyte to red blood cell ratio. 

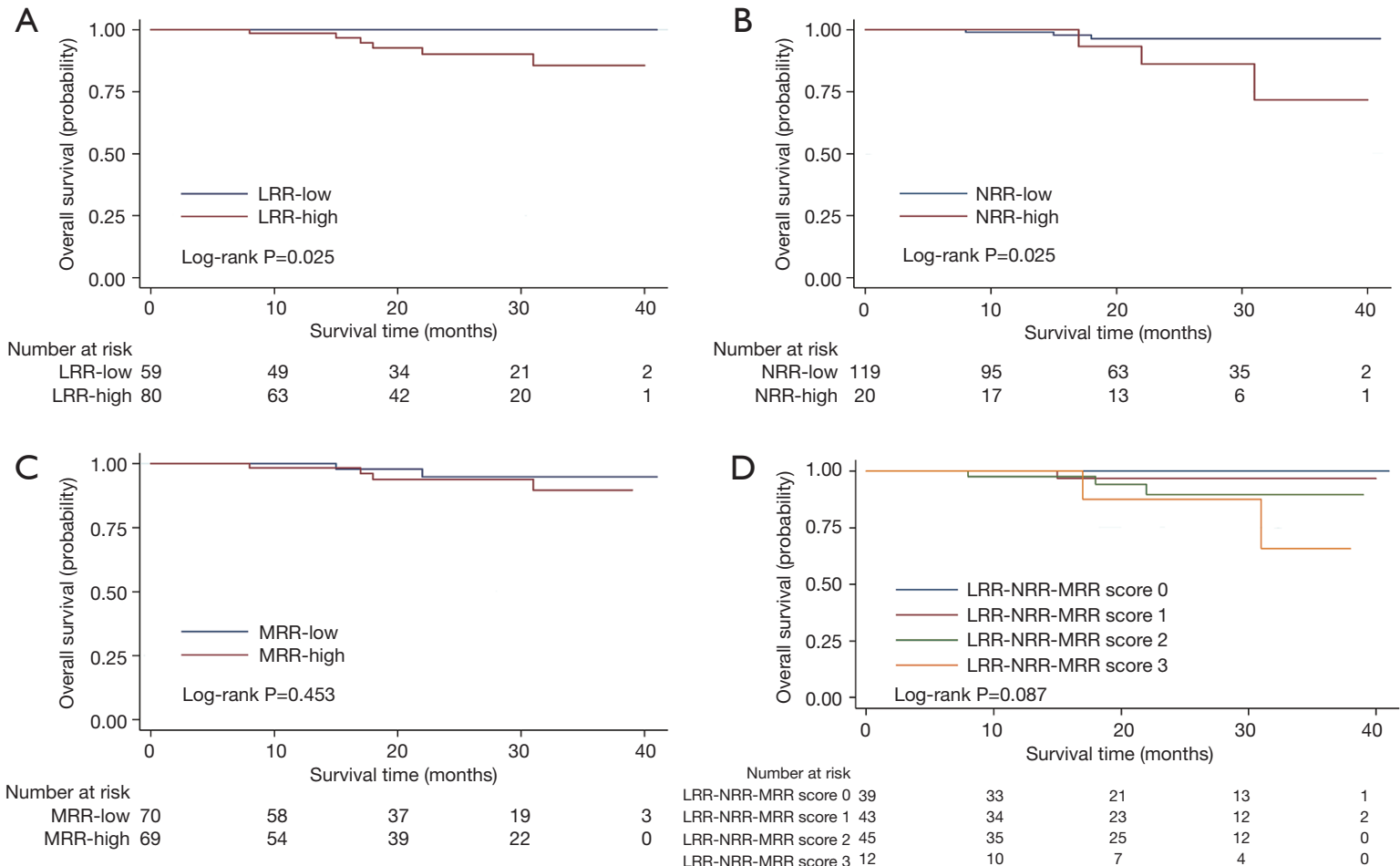

Figure 3 The Kaplan-Meier survival curve for overall survival in the locally advanced breast cancer patients. (A) Analysis by LRR; (B) analysis by NRR; (C) analysis by MRR; (D) analysis by the LRR-NRR-MRR score. LRR, lymphocyte to red blood cell ratio; NRR, neutrophil to red blood cell ratio; MRR, monocyte to red blood cell ratio.

Table 2 Cox Proportional Hazards Models: multivariate survival analysis of LRR-NRR-MRR score and disease-free survival, overall survival

\begin{tabular}{|c|c|c|c|c|c|c|c|}
\hline Variable & Categories & \multicolumn{3}{|c|}{ Disease-free survival } & \multicolumn{3}{|c|}{ Overall survival } \\
\hline LRR-NRR-MRR score system & 3 vs. 2 vs. 1 vs. 0 & 3.318 & $1.601-6.876$ & $0.001^{*}$ & 3.160 & $1.030-9.696$ & $0.044^{*}$ \\
\hline Clinical T stage & T3 T4 vs. T1 T2 & 2.824 & $0.940-8.481$ & 0.064 & 1.735 & $0.298-10.09$ & 0.540 \\
\hline ER status & Positive vs. negative & 1.533 & $0.345-6.802$ & 0.574 & 1.368 & $0.110-16.98$ & 0.808 \\
\hline Ki67 index & $>20$ vs. $\leq 20$ & 18.190 & $2.264-146.1$ & $0.006^{*}$ & 3.110 & $0.341-28.38$ & 0.314 \\
\hline HER2 status & Positive vs. negative & 1.305 & $0.472-3.610$ & 0.608 & 1.258 & $0.185-8.548$ & 0.814 \\
\hline BMI & $\geq 23.5$ vs. $<23.5$ & 1.167 & $0.386-3.531$ & 0.784 & 0.669 & $0.107-4.189$ & 0.668 \\
\hline
\end{tabular}

Italic values indicate statistically significant. * means $\mathrm{P}<0.05$. HR, hazard ratio; $\mathrm{Cl}$, confidence interval; HER2, human epidermal growth factor receptor 2; ER, Estrogen receptors; PR, Progesterone receptors; HER2, human epidermal growth factor receptor 2; MRR, monocyte to red blood cell ratio; BMI, body mass index.

tumor. LRR, MRR and NRR were assumed as the intensity indicator of the lymphocyte, monocyte and neutrophil in this study. We explored the predictive and prognostic value of each indicator and the combination of the three in the neoadjuvant therapy patients. As far as we knew, it was the first time to mention that pretreatment lower LRR was the favorable factor for the response to the neoadjuvant therapy. The lower LRR, NRR, MRR were identified as independent prognostic parameters for DFS of patients. Moreover, the LRR-MRR-NRR score which reflected 
the comprehensive status of lymphocyte, monocyte and neutrophil was also the first reported. The LRR-MRRNRR score was a predictor for neoadjuvant chemotherapy response and also an independently prognostic factor for DFS and OS.

The prognostic role of peripheral lymphocytes was still uncertain. In triple-negative breast cancer patients, the peripheral lymphocyte predicted the mortality (10). However, it was not proven in a study included all subtype of breast cancer (11). Lymphocyte was proved as a double-edged sword. Whether it did good or harm to tumor might depend on which kinds of lymphocytes accounted for a dominant position. It was reported elevated immunosuppressive phenotypes of peripheral lymphocytes after surgery was related with poor prognosis (12). Patients with locally advanced breast cancer had significantly more circulating T-regulatory (Tregs) than the health women. The good pathological response group showed relatively lower level of Tregs (13). Therefore, lower immunosuppressive lymphocyte might be related with better response and prognosis. However, the absolute lymphocyte count might be influenced by individual difference like nutrition status. Thus, we postulated using red blood cell as normalization could balance the individual variability and LRR could reflect the intensity role of lymphocyte. As a result, we found that pretreatment lower LRR might predict the better response of treatment and the prognosis for the patients treated with neoadjuvant chemotherapy.

Our results showed that Low NRR contributed to the better clinical outcome after neoadjuvant chemotherapy. Previous studies showed that lower NLR were also related with higher pCR $(6,8)$ and better prognosis $(9,14)$. Meanwhile, Low baseline peripheral neutrophil count was related with longer DFS (15). These studies supported our results. However, if both neutrophil and lymphocyte were low, NLR might not change. As our parameter separated neutrophil and lymphocyte, it partially indicated that neutrophils really played an important role both in the sensitivity of NAC and prognosis. It is proposed that neutrophils can engage in fostering immunosuppressive environment through production of cytokines or cooperation with other immune cells (16). For instance, the subsets of peripheral blood neutrophils (polymorphonuclear leukocytes) could release CCL2 which was related with heavy tumor load and poor prognosis in hepatocellular carcinoma patients (17). Tumor-associated neutrophils were suggested to recruit Treg into tumors via secretion of CCL17 and further impaired antitumor immune activity (18). The immunosuppressive microenvironment could facilitate tumor growth and induce resistance of antitumor therapy. The effect of weekly paclitaxel and cisplatin largely depended on inducing the antitumor immunity (19). Thus, high NRR which represented neutrophil intensity might be related with immunosuppression which caused worse effect of this regimen and predict the sensitivity and prognosis of patients.

It was demonstrated that peripheral absolute monocyte count (AMC) was an unfavorable prognostic factor for OS of early breast cancer patients (11). Recently, it was not found relate with both DFS and OS of locally advanced breast cancer patients received neoadjuvant chemotherapy (9). Therefore, MRR might be regarded as adjusted monocyte by the red blood cell and expected as a better index than the AMC. In our study, AMC also didn't reveal the significant relationship with the DFS and OS in locally advanced breast cancer (data not show). But, the MRR showed tightly related the poor DFS. In the tumor microenvironment, inflammatory monocytes could promote the extravasation of tumor cells which were correlated with poor prognosis and metastatic disease in breast cancer (20). Moreover, tumorassociated macrophages in the tissues (TAMs) which come from the circulating monocytes could produce abundant antiinflammatory factors and facilitate tumor development (21). By including three biomarkers of LRR, NRR and MRR, the LRR-NRR-MRR score covered all subsets of white blood cell and reflected the immune responses mediated by the lymphocyte, monocyte and neutrophil concurrently. The body as a whole changed, only one kind of immune cell changed is impossible. Thus, the LRR-NRR-MRR score turned out to be much better than the single ratio for the prognostic performance.

The limitations of this study were as following. First, this was a retrospective explore analysis based on two prospective clinical trials. However, the information of clinical and pathological characteristics was collected prospectively and documented in detail. The follow-up of patients was prospective. Second, the sample size was small. While we can already see the statistical difference of the biomarkers. Additional large independent validation set is needed to verify new biomarkers.

\section{Conclusions}

In summary, LRR-NRR-MRR score could be identified as a predictive biomarker for the therapeutic effect of 
neoadjuvant therapy and the independent prognostic factor for both DFS and OS for locally advanced breast cancer.

\section{Acknowledgments}

Funding: This work was supported by the National Natural Science Foundation of China (grant numbers 81172505 , 81302302), the Shanghai Municipal Commission of Health and Family Planning (grant number 201640006), the Clinical Research Plan of SHDC (grant number 16CR3065B, grant number SHDC 12016231), the Nurturing Fund of Renji Hospital (grant number PYMDT-002), the Shanghai "Rising Stars of Medical Talent" Youth Development Program Youth Medical Talents - Specialist Program (grant number 2018-15), and the Science and Technology Commission of Shanghai Municipality (grant number 14411950202, grant number 15JC1402700). The funders had no role in study design, data collection and analysis, decision to publish, or preparation of the manuscript.

\section{Footnote}

Conflicts of Interest: The authors have no conflicts of interest to declare.

Ethical Statement: All procedures performed in studies involving human participants were in accordance with the ethical standards of the institutional and/or national research committee and with the 1964 Helsinki declaration and its later amendments or comparable ethical standards. The study was approved by the Institutional Review Board (IRB) of Renji Hospital, School of Medicine, Shanghai Jiao Tong University (IRB approval number: [2017]088). Written informed consent was provided by all patients. Clinical trials registry: NCT, NCT 02221999, Registered August 21, 2014, https://clinicaltrials.gov/ct2/show/NCT02221999; NCT, NCT02879513, Registered August 25, 2016, https:// clinicaltrials.gov/ct2/show/NCT02879513. The authors are accountable for all aspects of the work in ensuring that questions related to the accuracy or integrity of any part of the work are appropriately investigated and resolved.

\section{References}

1. Siegel RL, Miller KD, Jemal A. Cancer statistics, 2018. CA Cancer J Clin 2018;68:7-30.

2. Fan L, Strasser-Weippl K, Li JJ, et al. Breast cancer in
China. Lancet Oncol 2014;15:e279-89.

3. Ataseven B, von Minckwitz G. The Impact of Neoadjuvant Treatment on Surgical Options and Outcomes. Ann Surg Oncol 2016;23:3093-9.

4. McMillan DC. Systemic inflammation, nutritional status and survival in patients with cancer. Curr Opin Clin Nutr Metab Care 2009;12:223-6.

5. $\mathrm{Xu} \mathrm{J}, \mathrm{Ni} \mathrm{C}, \mathrm{Ma} \mathrm{C}$, et al. Association of neutrophil/ lymphocyte ratio and platelet/lymphocyte ratio with $\mathrm{ER}$ and $\mathrm{PR}$ in breast cancer patients and their changes after neoadjuvant chemotherapy. Clin Transl Oncol 2017;19:989-96.

6. Chen Y, Chen K, Xiao X, et al. Pretreatment neutrophilto-lymphocyte ratio is correlated with response to neoadjuvant chemotherapy as an independent prognostic indicator in breast cancer patients: a retrospective study. BMC Cancer 2016;16:320.

7. Ni XJ, Zhang XL, Ou-Yang QW, et al. An elevated peripheral blood lymphocyte-to-monocyte ratio predicts favorable response and prognosis in locally advanced breast cancer following neoadjuvant chemotherapy. PLoS One 2014;9:e111886.

8. Asano Y, Kashiwagi S, Onoda N, et al. Predictive Value of Neutrophil/Lymphocyte Ratio for Efficacy of Preoperative Chemotherapy in Triple-Negative Breast Cancer. Ann Surg Oncol 2016;23:1104-10.

9. Marín Hernández C, Pinero Madrona A, Gil Vazquez PJ, et al. Usefulness of lymphocyte-to-monocyte, neutrophil-tomonocyte and neutrophil-to-lymphocyte ratios as prognostic markers in breast cancer patients treated with neoadjuvant chemotherapy. Clin Transl Oncol 2018;20:476-83.

10. Afghahi A, Purington N, Han SS, et al. Higher Absolute Lymphocyte Counts Predict Lower Mortality from EarlyStage Triple-Negative Breast Cancer. Clin Cancer Res 2018;24:2851-8.

11. Wen J, Ye F, Huang X, et al. Prognostic Significance of Preoperative Circulating Monocyte Count in Patients With Breast Cancer: Based on a Large Cohort Study. Medicine (Baltimore) 2015;94:e2266.

12. Fu G, Miao L, Wang M, et al. The Postoperative Immunosuppressive Phenotypes of Peripheral T Helper Cells Are Associated with Poor Prognosis of Breast Cancer Patients. Immunol Invest 2017;46:647-62.

13. Verma C, Eremin JM, Robins A, et al. Abnormal T regulatory cells (Tregs: FOXP3+, CTLA-4+), myeloidderived suppressor cells (MDSCs: monocytic, granulocytic) and polarised T helper cell profiles (Th1, Th2, Th17) in women with large and locally advanced breast cancers 
undergoing neoadjuvant chemotherapy (NAC) and surgery: failure of abolition of abnormal treg profile with treatment and correlation of treg levels with pathological response to NAC. J Transl Med 2013;11:16.

14. Ethier JL, Desautels D, Templeton A, et al. Prognostic role of neutrophil-to-lymphocyte ratio in breast cancer: a systematic review and meta-analysis. Breast Cancer Res 2017;19:2.

15. Qian Y, Tao J, Li X, et al. Peripheral inflammation/ immune indicators of chemosensitivity and prognosis in breast cancer patients treated with neoadjuvant chemotherapy. Onco Targets Ther 2018;11:1423-32.

16. Liang W, Ferrara N. The Complex Role of Neutrophils in Tumor Angiogenesis and Metastasis. Cancer Immunol Res 2016;4:83-91.

17. Tsuda Y, Fukui H, Asai A, et al. An immunosuppressive

Cite this article as: Wang Y, Wang H, Yin W, Lin Y, Zhou L, Sheng X, Xu Y, Sha R, Lu J. Novel lymphocyte to red blood cell ratio (LRR), neutrophil to red blood cell ratio (NRR), monocyte to red blood cell ratio (MRR) as predictive and prognostic biomarkers for locally advanced breast cancer. Gland Surg 2019;8(6):627-635. doi: 10.21037/gs.2019.10.10 subtype of neutrophils identified in patients with hepatocellular carcinoma. J Clin Biochem Nutr 2012;51:204-12.

18. Mishalian I, Bayuh R, Eruslanov E, et al. Neutrophils recruit regulatory $\mathrm{T}$-cells into tumors via secretion of CCL17--a new mechanism of impaired antitumor immunity. Int J Cancer 2014;135:1178-86.

19. Chang CL, Hsu Y'T, Wu CC, et al. Dose-dense chemotherapy improves mechanisms of antitumor immune response. Cancer Res 2013;73:119-27.

20. Qian BZ, Li J, Zhang H, et al. CCL2 recruits inflammatory monocytes to facilitate breast-tumour metastasis. Nature 2011;475:222-5.

21. Tang X. Tumor-associated macrophages as potential diagnostic and prognostic biomarkers in breast cancer. Cancer Lett 2013;332:3-10. 
Supplementary

Table S1 Univariate logistic regression models of LRR, NRR, MRR, LRR-NRR-MRR score, clinical pathological characteristic and pathological complete rate of neoadjuvant therapy

\begin{tabular}{|c|c|c|c|c|}
\hline \multirow{2}{*}{ Variable } & \multirow{2}{*}{ Categories } & \multicolumn{3}{|c|}{ Total pCR rate } \\
\hline & & OR & $95 \% \mathrm{Cl}$ & $P$ \\
\hline LRR & High vs. low & 0.564 & $0.269-1.183$ & 0.129 \\
\hline NRR & High vs. low & 0.391 & $0.108-1.417$ & 0.153 \\
\hline MRR & High vs. low & 0.668 & $0.319-1.401$ & 0.286 \\
\hline LRR-NRR-MRR score & 2,3 vs. 0,1 & 0.661 & $0.443-0.986$ & $0.042^{*}$ \\
\hline Clinical T stage & T3 T4 vs. T1 T2 & 0.667 & $0.319-1.394$ & 0.281 \\
\hline ER status & Positive vs. negative & 0.201 & $0.090-0.449$ & $0.000^{*}$ \\
\hline PR status & Positive vs. negative & 0.417 & $0.174-0.996$ & $0.049^{*}$ \\
\hline Ki67 index & $>20$ vs. $\leq 20$ & 3.660 & $1.579-8.480$ & $0.002^{*}$ \\
\hline HER2 status & Positive vs. negative & 3.333 & $1.552-7.159$ & $0.002^{*}$ \\
\hline BMI & $\geq 23.5$ vs. $<23.5$ & 0.418 & $0.193-0.903$ & $0.026^{*}$ \\
\hline
\end{tabular}

Italic values indicate statistically significant. * means $\mathrm{P}<0.05 . \mathrm{Cl}$, confidence interval; HER2, human epidermal growth factor receptor 2; ER, Estrogen receptors; PR, Progesterone receptors LRR, lymphocyte to red blood cell ratio; NRR, neutrophil to red blood cell ratio; MRR, monocyte to red blood cell ratio; BMI, body mass index.

Table S2 Multivariate logistic regression models of LRR, NRR, MRR, LRR-NRR-MRR score and pathological complete response of neoadjuvant therapy

\begin{tabular}{|c|c|c|c|c|}
\hline \multirow{2}{*}{ Variable } & \multirow{2}{*}{ Categories } & \multicolumn{3}{|c|}{ pCR } \\
\hline & & OR & $95 \% \mathrm{Cl}$ & $\mathrm{P}$ \\
\hline LRR & High vs. low & 0.239 & $0.085-0.672$ & $0.007^{*}$ \\
\hline NRR & High vs. low & 0.437 & $0.101-1.889$ & 0.268 \\
\hline MRR & High vs. low & 0.749 & $0.305-1.836$ & 0.527 \\
\hline LRR-NRR-MRR score & 2,3 vs. 0,1 & 0.593 & $0.369-0.954$ & $0.031^{*}$ \\
\hline
\end{tabular}

Italic values indicate statistically significant. * means $\mathrm{P}<0.05 . \mathrm{Cl}$, confidence interval; $\mathrm{pCR}$, pathological complete response; LRR, lymphocyte to red blood cell ratio; NRR, neutrophil to red blood cell ratio; MRR, monocyte to red blood cell ratio.

Table S3 Univariate logistic regression models of LRR-NRR-MRR score and pathological complete response in different subtype of breast cancer patients

\begin{tabular}{|c|c|c|c|c|}
\hline \multirow{2}{*}{ Variable } & \multirow{2}{*}{ No. } & \multicolumn{3}{|c|}{$\mathrm{pCR}$} \\
\hline & & OR & $95 \% \mathrm{Cl}$ & $P$ \\
\hline HR positive HER2 negative & 69 & 0.764 & $0.370-1.575$ & 0.465 \\
\hline HR positive HER2 positive & 49 & 0.451 & $0.233-0.871$ & $0.018^{*}$ \\
\hline HR negative HER2 positive & 9 & 0.866 & $0.179-4.184$ & 0.858 \\
\hline HR negative HER2 negative & 12 & 1.061 & $0.330-3.405$ & 0.921 \\
\hline HR negative & 21 & 0.894 & $0.376-2.129$ & 0.801 \\
\hline HR positive & 118 & 0.591 & $0.368-0.947$ & $0.029^{*}$ \\
\hline HER2 negative & 81 & 0.922 & $0.521-1.632$ & 0.781 \\
\hline HER2 positive & 58 & 0.485 & $0.267-0.881$ & $0.018^{*}$ \\
\hline
\end{tabular}

Italic values indicate statistically significant. * means $\mathrm{P}<0.05 . \mathrm{Cl}$, confidence interval; $\mathrm{pCR}$, pathological complete response; LRR, lymphocyte to red blood cell ratio; NRR, neutrophil to red blood cell ratio; MRR, monocyte to red blood cell ratio. 
Table S4 Cox Proportional Hazards Models: multivariate survival analysis of LRR and disease-free survival, overall survival

\begin{tabular}{|c|c|c|c|c|c|c|c|}
\hline \multirow{2}{*}{ Variable } & \multirow{2}{*}{ Categories } & \multicolumn{3}{|c|}{ Disease-free survival } & \multicolumn{3}{|c|}{ Overall survival } \\
\hline & & HR & $95 \% \mathrm{Cl}$ & $\mathrm{P}$ & $\mathrm{HR}$ & $95 \% \mathrm{Cl}$ & $\mathrm{P}$ \\
\hline LRR & High vs. low & 4.447 & $1.236-16.00$ & $0.022^{*}$ & $5.71 \mathrm{E}+15$ & 0 & 1 \\
\hline Clinical T stage & T3 T4 vs. T1 T2 & 2.759 & $0.878-8.667$ & 0.082 & 2.990 & $0.499-17.92$ & 0.231 \\
\hline ER status & Positive vs. negative & 1.191 & $0.272-5.210$ & 0.816 & 1.322 & $0.076-22.99$ & 0.848 \\
\hline PR status & Positive vs. negative & 1.089 & $0.219-5.401$ & 0.917 & 0.328 & $0.019-5.626$ & 0.442 \\
\hline Ki67 index & $>20$ vs. $\leq 20$ & 10.79 & $1.395-83.47$ & $0.023^{*}$ & 2.779 & $0.319-24.25$ & 0.355 \\
\hline HER2 status & Positive vs. negative & 0.975 & $0.352-2.696$ & 0.960 & 0.597 & $0.106-3.377$ & 0.560 \\
\hline BMI & $\geq 23.5$ vs. $<23.5$ & 1.195 & $0.392-3.644$ & $0.022^{*}$ & 0.467 & $0.081-2.686$ & 0.394 \\
\hline
\end{tabular}

Italic values indicate statistically significant. ${ }^{*}$ means $\mathrm{P}<0.05$. HR, hazard ratio; $\mathrm{Cl}$, confidence interval; HER2, human epidermal growth factor receptor 2; ER, Estrogen receptors; PR, Progesterone receptors; HER2, human epidermal growth factor receptor 2; LRR, lymphocyte to red blood cell ratio; BMI, body mass index.

Table S5 Cox Proportional Hazards Models: multivariate survival analysis of NRR and disease-free survival, overall survival

\begin{tabular}{|c|c|c|c|c|c|c|c|}
\hline \multirow{2}{*}{ Variable } & \multirow{2}{*}{ Categories } & \multicolumn{3}{|c|}{ Disease-free survival } & \multicolumn{3}{|c|}{ Overall survival } \\
\hline & & $\mathrm{HR}$ & $95 \% \mathrm{Cl}$ & $\mathrm{P}$ & $\mathrm{HR}$ & $95 \% \mathrm{Cl}$ & $\mathrm{P}$ \\
\hline NRR & High vs. low & 4.524 & $1.191-17.18$ & $0.027^{*}$ & 6.648 & $0.882-50.11$ & 0.066 \\
\hline Clinical T stage & T3 T4 vs. T1 T2 & 1.727 & $0.571-5.432$ & 0.333 & 1.205 & $0.175-8.308$ & 0.850 \\
\hline ER status & Positive vs. negative & 1.511 & $0.420-5.432$ & 0.527 & 1.219 & $0.147-10.09$ & 0.854 \\
\hline PR status & Positive vs. negative & 0.862 & $0.224-3.321$ & 0.829 & 0.260 & $0.032-2.137$ & 0.210 \\
\hline Ki67 index & $>20$ vs. $\leq 20$ & 25.00 & $2.340-267.2$ & $0.008^{*}$ & 3.416 & $0.355-32.84$ & 0.287 \\
\hline HER2 status & Positive vs. negative & 1.417 & $0.511-3.931$ & 0.503 & 1.034 & $0.171-6.246$ & 0.971 \\
\hline BMI & $\geq 23.5$ vs. $<23.5$ & 1.553 & $0.522-4.620$ & 0.428 & 0.742 & $0.117-4.699$ & 0.751 \\
\hline
\end{tabular}

Italic values indicate statistically significant. * means $\mathrm{P}<0.05$. HR, hazard ratio; Cl, confidence interval; HER2, human epidermal growth factor receptor 2; ER, Estrogen receptors; PR, Progesterone receptors; HER2, human epidermal growth factor receptor 2; NRR, neutrophil to red blood cell ratio; BMI, body mass index.

Table S6 Cox Proportional Hazards Models: multivariate survival analysis of MRR and disease-free survival, overall survival

\begin{tabular}{|c|c|c|c|c|c|c|c|}
\hline \multirow{2}{*}{ Variable } & \multirow{2}{*}{ Categories } & \multicolumn{3}{|c|}{ Disease-free survival } & \multicolumn{3}{|c|}{ Overall survival } \\
\hline & & $\mathrm{HR}$ & $95 \% \mathrm{Cl}$ & $P$ & $\mathrm{HR}$ & $95 \% \mathrm{Cl}$ & $P$ \\
\hline MRR & High vs. low & 3.940 & $1.201-12.93$ & $0.024^{*}$ & 2.196 & $0.352-13.72$ & 0.400 \\
\hline Clinical T stage & T3 T4 vs. T1 T2 & 2.771 & $0.908-8.455$ & 0.073 & 2.439 & $0.443-13.42$ & 0.305 \\
\hline ER status & Positive vs. negative & 1.468 & $0.400-5.391$ & 0.563 & 1.011 & $0.131-7.792$ & 0.992 \\
\hline PR status & Positive vs. negative & 0.730 & $0.172-3.096$ & 0.670 & 0.303 & $0.040-2.313$ & 0.249 \\
\hline Ki67 index & $>20$ vs. $\leq 20$ & 13.741 & $1.744-108.2$ & $0.013^{*}$ & 3.105 & $0.351-27.46$ & 0.308 \\
\hline HER2 status & Positive vs. negative & 1.461 & $0.531-4.020$ & 0.463 & 0.908 & $0.151-5.445$ & 0.916 \\
\hline $\mathrm{BMI}$ & $\geq 23.5$ vs. $<23.5$ & 0.967 & $0.332-2.815$ & 0.950 & 0.407 & $0.073-2.288$ & 0.308 \\
\hline
\end{tabular}

Italic values indicate statistically significant. * means $\mathrm{P}<0.05$. HR, hazard ratio; Cl, confidence interval; HER2, human epidermal growth factor receptor 2; ER, Estrogen receptors; PR, Progesterone receptors; HER2, human epidermal growth factor receptor 2; MRR, monocyte to red blood cell ratio; BMI, body mass index. 